\title{
Gauss decomposition of trigonometric R-matrices
}

\author{
S.M.Khoroshkin \\ ITEP, Bol'shaya Cheremushkinskayas 25, \\ 117259 Moscow, Russia \\ A.A.Stolin \\ Department of Mathematics, Royal Institute of Technology, \\ S-10044 Stockholm, Sweden \\ V.N.Tolstoy \\ Institute of Nuclear Physics, Moscow State University, \\ 119899 Moscow
}

\begin{abstract}
The general formula for the universal R-matrix for quantized nontwisted affine algebras by Khoroshkin and Tolstoy is applied for zero central charge highest weight modules of quantized affine algebras. It is shown how the universal $R$-matrix produces the Gauss decomposition of trigonomitric $R$-matrix in tensor product of these modules. Explicit calculations for the simplest case of $A_{1}^{(1)}$ are presented. As a consequence new formulas for the trigonometric $R$-matrix with a parameter in tensor product of $U_{q}\left(s l_{2}\right)$-Verma modules are obtained.
\end{abstract}

\section{Introduction}

The most known technique for calculating trigonometric solutions of the Yang-Baxter equation (with a parameter) looks as follows.

One can fix a quantum deformation $U_{q}(g)$ of the universal enveloping algebra of a simple Lie algebra $g$ and write down the trigonometric solutions of the Yang-Baxter equation in fundamental representations of $U_{q}(g)$ which are all known (see [J]1). Then the $R$-matrices in other finite-dimensional representations of $U_{q}(g)$ can be obtained by so called fusion procedure [KS], [KRS], [J2] which is based on the analysis of $q$-analogs of the Young symmetrizes in tensor productes of fundamental representations [Ch1, CCh2].

In alternative approach one can apply the universal $R$-matrix [Dr1], [Dr2] to tensor product of concrete representations. For the purpose of parametric trigonometric solutions of the Yang-Baxter equation one should use the universal $R$-matrix for quantized affine algebras. An explicit formula for universal $R$-matrix for quantized nontwisted affine algebras was found in [KT2]. It has multiplicative form and can be presented in terms of infinite product over the set of all positive root of the corresponding affine algebra with the factors being some $(q)$-exponential functions of $U_{q}(\widehat{g} \otimes \widehat{g})$. The complicated form 
of these infinite products restricts the number of applications of the universal $R$-matrix. However, some low dimensional examples are given in [KT2], [KT3], [ZG].

The aim of this paper is to remove some psychological obstacles and to demonstrate the technique of working with the universal $R$-matrix in tensor product of some zero charge highest weight representations of quantized affine algebras.

First we note that due to the general structure of normal (or convex in terminology, suggested by V.G.Kac) ordering of positive roots of affine Lie algebra the universal $R$-matrix can be naturally divided into three parts which produce generalized Gauss decomposition of $R$-matrix in concrete representations.

Next we demonstrate in $A_{1}^{(1)}$ case how to calculate these three parts in tensor products of the representations, induced from Verma or finite dimensional modules of finite $U_{q}\left(s l_{2}\right)$ (more exactly, for corresponding evaluation representations of $U_{q}^{\prime}\left(\widehat{s l}_{2}\right)$ ). Moreover, we reexpress the entries of the Gauss decomposition in terms of $U_{q}\left(s l_{2}\right) \otimes U_{q}\left(s l_{2}\right)$ generators with coefficients being degenerated basic hypergeometric functions of Cartan elements. As a consequence we obtain the general form of Gauss decomposition of trigonometric $R$-matrix in tensor product of $s l_{2}$-Verma modules (and of finite dimensional modules as factors of Verma modules). These examples show that a field of applications of the universal $R$ - matrix is not covered by fusion technique.

It is not useless to look at the structure of presented Gauss factors of trigonometric $R$-matrix. The nilpotent parts have very simple $q$-exponential structure with coeffitients being rational function of spectral parameter usual for trigonometric $R$-matrix. The diagonal component can be divided into three different parts. The first gives the scalar factor [Dr1], KT2 being the solution of one-dimensional q-difference Knizhnik-Zamolodchikov equation [FR]. The two others have rational coefficients for finite dimensional representations but one of them is not rational for Verma modules with noninteger highest weights. In this case the $R$-matrix cannot be expressed in terms of rational functions of spectral parameter.

Acknowlegements. The authors are thankful to the Academy of Science of Sweden for the hospitality of Royal Institute of Technologies where the first steps of the work were done. The first and the third authors are also especially thankful to Professor C.M.Ringel for the kindly atmosphere in SFB 343 of Mathematical Departement of Bielefeld University where the work was completed.

\section{Notations}

Let $\hat{g}$ be nontwisted affine Lie algebra with symmetrizable Cartan matrix $A=\left(a_{i j}\right)$ $\left(A^{\text {sym }}=\left(a_{i j}^{\text {sym }}\right)\right.$ is the correspoding symmetrical matrix $)$ and let $\Pi=\left\{\alpha_{0}, \alpha_{1}, \ldots, \alpha_{r}\right\}$ be a system of simple roots for $\hat{g}$. We assume that the roots $\Pi_{0}=\left\{\alpha_{1}, \alpha_{2}, \ldots, \alpha_{r}\right\}$ generate the system $\Delta_{+}(g)$ of positive roots of the corresponding finite-dimensional Lie algebra $g$. The quantum deformation $U_{q}^{\prime}(\hat{g})$ is an associative algebra over the ring of formal power series $\mathbf{C}[[q-1]]$ with generators $e_{ \pm \alpha_{i}}, k_{\alpha_{i}}^{ \pm 1}=q^{ \pm h_{\alpha_{i}}}, \quad(i=1,2, \ldots, r)$, and the defining relations

$$
\left[k_{\alpha_{i}}^{ \pm 1}, k_{\alpha_{j}}^{ \pm 1}\right]=0, \quad k_{\alpha_{i}} e_{ \pm \alpha_{j}}=q^{ \pm\left(\alpha_{i}, \alpha_{j}\right)} e_{ \pm \alpha_{j}} k_{\alpha_{i}}
$$




$$
\begin{gathered}
{\left[e_{\alpha_{i}}, e_{-\alpha_{j}}\right]=\delta_{i j} \frac{k_{\alpha_{i}}-k_{\alpha_{i}}^{-1}}{q-q^{-1}}} \\
\left(a d_{q^{\prime}} e_{ \pm \alpha_{i}}\right)^{1-a_{i j}} e_{ \pm \alpha_{j}}=0 \quad \text { for } i \neq j, q^{\prime}=q, q^{-1}
\end{gathered}
$$

where $\left(a d_{q} e_{\alpha}\right) e_{\beta}$ is a q-commutator:

$$
\left(a d_{q} e_{\alpha}\right) e_{\beta} \equiv\left[e_{\alpha}, e_{\beta}\right]_{q}=e_{\alpha} e_{\beta}-q^{(\alpha, \beta)} e_{\beta} e_{\alpha}
$$

and $(\alpha, \beta)$ is a scalar product of the roots $\alpha$ and $\beta:\left(\alpha_{i}, \alpha_{j}\right)=a_{i j}^{s y m}$.

The condition $c=0$ is also imposed in $U^{\prime}{ }_{q}(\hat{g})$, where $c$ can be defined by the relation $q^{c}=k_{\alpha_{0}}^{n_{0}} k_{\alpha_{1}}^{n_{1}} \cdots k_{\alpha_{r}}^{n_{r}}$ with $n_{0}=\sum_{i \geq 1} n_{i}, \sum_{i \geq 1} n_{i} \alpha_{i}=\theta, \theta$ being the highest root of $\Delta_{+}(g)$.

We define a comultiplication in $U^{\prime}{ }_{q}(\hat{g})$ by the formulas

$$
\begin{gathered}
\Delta\left(k_{\alpha_{i}}\right)=k_{\alpha_{i}} \otimes k_{\alpha_{i}}, \\
\Delta\left(e_{\alpha_{i}}\right)=e_{\alpha_{i}} \otimes 1+k_{\alpha_{i}}^{-1} \otimes e_{\alpha_{i}}, \Delta\left(e_{-\alpha_{i}}\right)=e_{-\alpha_{i}} \otimes k_{\alpha_{i}}+1 \otimes e_{-\alpha_{i}}
\end{gathered}
$$

We denote by a symbol $\left(^{*}\right)$ an antiinvolution in $U_{q}(\hat{g})$, defined as $\left(k_{\alpha_{i}}\right)^{*}=k_{\alpha_{i}}^{-1}$, $\left(e_{ \pm \alpha_{i}}\right)^{*}=e_{\mp \alpha_{i}}, \quad(q)^{*}=q^{-1}$.

An algebra $U_{q}(\hat{g})$ is an associative algebra over the ring of formal power series $\mathbf{C}[[q-1]]$ with generators $e_{ \pm \alpha_{i}}, \quad k_{\alpha_{i}}^{ \pm 1}=q^{ \pm h_{\alpha_{i}}},(i=1,2, \ldots, r)$; $d$, with the relations ( 1.1) $-($ (1.3) and

$$
\left[d, k_{\alpha_{i}}^{ \pm 1}\right]=0, \quad\left[d, e_{ \pm \alpha_{i}}\right]= \pm \delta_{i, 0} e_{ \pm \alpha_{i}}
$$

Algebra $U_{q}(\hat{g})$ is also a Hopf algebra with the comultiplication ( 1.5)-( 1.6) and

$$
\Delta(d)=d \otimes 1+1 \otimes d
$$

We use also the following standard notations:

$$
\begin{gathered}
\exp _{q}(x):=1+x+\frac{x^{2}}{(2)_{q} !}+\ldots+\frac{x^{n}}{(n)_{q} !}+\ldots=\sum_{n \geq 0} \frac{x^{n}}{(n)_{q}} \\
(a)_{q}:=\frac{q^{a}-1}{q-1}, \quad[a]_{q}:=\frac{q^{a}-q^{-a}}{q-q^{-1}}, \quad q_{\alpha}:=q^{-(\alpha, \alpha)}
\end{gathered}
$$

and

$$
k_{\gamma}=q^{h_{\gamma}}=k_{\alpha_{0}}^{n_{0}} \cdots k_{\alpha_{r}}^{n_{r}}
$$

if $\gamma=n_{0} \alpha_{0}+\ldots n_{r} \alpha_{r}$.

\section{Cartan-Weyl basis and the universal R-matrix for quantum affine algebras}

Let $\Delta_{+}:=\Delta_{+}(\hat{g})$ be the system of all positive roots of $\hat{g}$ with respect to $\Pi$.

It turns out that a procedure of the construction of the quantum Cartan-Weyl basis has to be in agreement with the choise of normal ordering in the root system $\Delta_{+}$. Let us recall the definition of normal order in $\Delta_{+}$AST. 
Definition 2.1 We say that the system $\Delta_{+}$is in normal (or convex) ordering if its roots are totally ordered in the following way: (i) all multiple roots follow each other in an arbitrary order; (ii) each nonsimple root $\alpha+\beta \in \Delta_{+}$, where $\alpha \neq \lambda \beta$ has to be posed between $\alpha$ and $\beta$.

Fix some normal ordering in $\Delta_{+}=\Delta_{+}^{r e} \cup \Delta_{+}^{i m}$, satisfying an additional condition:

$$
\alpha_{i}+n \delta<k \delta<\left(\delta-\alpha_{j}\right)+l \delta
$$

for any simple roots $\alpha_{i}, \alpha_{j} \in \Delta_{+}(g), l, n \geq 0, k>0$. Here $\delta$ is a minimal positive imaginary root. Apply the following inductive procedure for the construction of real root vectors $e_{ \pm \gamma}, \gamma \in \Delta_{+}^{r e}$, starting from simple root vectors of $\Delta_{+}$.

Let $\gamma \in \Delta_{+}^{r e}$ be a real root and $\alpha, \ldots, \gamma, \ldots, \beta$ be a minimal subset restricting $\gamma(\gamma=$ $\alpha+\beta)$. Then we set

$$
e_{\gamma}:=\left[e_{\alpha}, e_{\beta}\right]_{q}, \quad e_{-\gamma}:=\left[e_{-\beta}, e_{-\alpha}\right]_{q^{-1}}
$$

if $e_{ \pm \alpha}$ and $e_{ \pm \beta}$ have already been constructed.

When we get the imaginary root $\delta$, we stop for a moment and use the following formulas:

$$
\begin{gathered}
e_{\delta}^{(i)}=\varepsilon_{1}\left(\alpha_{i}\right)\left[e_{\alpha_{i}}, e_{\delta-\alpha_{i}}\right]_{q}, \\
e_{\alpha_{i}+m \delta}=\varepsilon_{n}\left(\alpha_{i}\right)\left(-\left[\left(\alpha_{i}, \alpha_{i}\right)\right]_{q}\right)^{-m}\left(a d e_{\delta}^{(i)}\right)^{m} e_{\alpha_{i}}, \\
e_{\delta-\alpha_{i}+m \delta}=\varepsilon_{n}\left(\alpha_{i}\right)\left(\left[\left(\alpha_{i}, \alpha_{i}\right)\right]_{q}\right)^{-m}\left(a d e_{\delta}^{(i)}\right)^{m} e_{\delta-\alpha_{i}}, \\
e_{m \delta}^{\prime(i)}=\varepsilon_{n}\left(\alpha_{i}\right)\left[e_{\alpha_{i}+(m-1) \delta}, e_{\delta-\alpha_{i}}\right]_{q},
\end{gathered}
$$

(for $m>0$ ), where $(a d x) y=[x, y]$ is a usual commutator, $\varepsilon_{n}\left(\alpha_{i}\right)=(-1)^{n \theta\left(\alpha_{i}\right)}$, and $\theta: \Pi_{0}=\left\{\alpha_{1}, \ldots, \alpha_{r}\right\} \rightarrow\{0,1\}$ is chosen in such a way that

$$
\left(\alpha_{i}, \alpha_{j}\right) \neq 0 \quad \longrightarrow \quad \theta\left(\alpha_{i}\right) \neq \theta\left(\alpha_{j}\right)
$$

Then we use the inductive procedure again to obtain other real root vectors $e_{\gamma+n \delta}$, $e_{\delta-\gamma+n \delta}, \gamma \in \Delta_{+}(g)$. We come to the end by defining imaginary root vectors $e_{n \delta}^{(i)}$ through intermediate vectors $e_{n \delta}^{\prime(i)}$ by means of the following (Schur) relations:

$$
\left(q-q^{-1}\right) E^{(i)}(z)=\log \left(1+\left(q-q^{-1}\right) E^{\prime(i)}(z)\right)
$$

where $E^{(i)}(z)$ and $E^{\prime(i)}(z)$ are generating functions for $e_{n \delta}^{(i)}$ and for $e_{n \delta}^{\prime(i)}$ :

$$
\begin{aligned}
E^{(i)}(z) & =\sum_{n \geq 1} e_{n \delta}^{(i)} z^{-n} \\
E^{\prime(i)}(z) & =\sum_{n \geq 1} e_{n \delta}^{\prime(i)} z^{-n} .
\end{aligned}
$$


The root vectors of negative roots are obtained by the Cartan involution $(*)$ :

$$
e_{-\gamma}=\left(e_{\gamma}\right)^{*}
$$

for $\gamma \in \Delta_{+}(\hat{g})$.

An explicit expression for the universal $R$-matrix $\mathcal{R}$ (for the definition see [Dr2]) for quantum nontwisted affine algebras was given in [KT2]. Namely, for a fixed normal ordering of $\Delta_{+}(\hat{g})$, satisfying (2.1), we can present $\mathcal{R}$ as follows:

$$
\mathcal{R}=\overline{\mathcal{R}}_{r e}^{+} \overline{\mathcal{R}}_{i m} \overline{\mathcal{R}}_{r e}^{-} \mathcal{K}
$$

Here $\mathcal{K}=q^{\sum_{i, j} d_{i j} h_{i} \otimes h_{j}+(c \otimes d+d \otimes c)}$, where $d_{i j}$ is an inverse to symmetrical Cartan matrix $\left(b_{i j}^{s y m}\right), i, j=1, \ldots r$ of underlying finite dimensional Lie algebra $g$,

$$
\overline{\mathcal{R}}_{r e}^{+}=\prod_{\gamma \in \Delta_{+}^{r e}, \gamma<\delta}^{\rightarrow} R_{\gamma}, \quad \overline{\mathcal{R}}_{r e}^{-}=\prod_{\gamma \in \Delta_{+}^{r e}, \gamma>\delta}^{\overrightarrow{ }} R_{\gamma}
$$

where

$$
R_{\gamma}=\exp _{q_{\gamma}}\left(a(\gamma) e_{\gamma} \otimes e_{-\gamma}\right),
$$

$a(\gamma)$ is $\mathbf{C}(q)$ - coefficient in the relation

$$
\left[e_{\gamma}, e_{-\gamma}\right]=\frac{k_{\gamma}-k_{\gamma}^{-1}}{a(\gamma)}
$$

and the order in the product (2.11) coincides with a chosen for the construction of the Cartan-Weyl basis normal ordering of $\Delta_{+}$, satisfying (2.1). Finally,

$$
\overline{\mathcal{R}}_{i m}=\exp \left(\sum_{n>0} c_{i, j}^{n} e_{n \delta}^{(i)} \otimes e_{-n \delta}^{(j)}\right)
$$

where $c_{i, j}^{n}$ is $\left(q-q^{-1}\right)$ times an inverse matrix to

$$
\frac{\left[n\left(\alpha_{i}, \alpha_{j}\right)\right]_{q}}{n} .
$$

One can rewrite the general expression (2.11) for the universal $R$-matrix in more symmetric form using the following property of an element $\mathcal{K}$ in (2.11) (see [KT1]):

$$
\begin{gathered}
\left(e_{\gamma} \otimes 1\right) \mathcal{K}=\mathcal{K}\left(e_{\gamma} \otimes k_{\gamma}^{-1}\right), \\
\left(1 \otimes e_{-\gamma}\right) \mathcal{K}=\mathcal{K}\left(k_{\gamma} \otimes e_{-\gamma}\right)
\end{gathered}
$$

for any root vector $e_{\gamma}, \gamma \in \Delta_{+}$.

Let us define the following elements $\xi_{ \pm \gamma}, \gamma \in \Delta_{+}^{r e}$ and $x_{k}^{(i)}, i=1, \ldots, r, k \in \mathbf{Z}$ :

$$
\begin{array}{clr}
\xi_{\gamma}=e_{\gamma}, \quad \xi_{-\gamma}=e_{-\gamma} & \text { for } \gamma<\delta, \\
\xi_{\gamma}=e_{\gamma} k_{\gamma}, \quad \xi_{-\gamma}=k_{\gamma}^{-1} e_{-\gamma} & \text { for } \gamma>\delta
\end{array}
$$




$$
x_{ \pm k}^{(i)}=e_{ \pm k \delta}^{(i)} \text { for } k>0, \quad x_{0}^{(i)}=h_{\alpha_{i}} .
$$

Here $\delta$ is again a minimal imaginary root.

In these notations the universal $R$-matrix for $U_{q}(\hat{g})$ can be written as

$$
\mathcal{R}=\mathcal{R}_{r e}^{+} \mathcal{R}_{i m} \mathcal{R}_{r e}^{-}
$$

where

$$
\begin{gathered}
\mathcal{R}_{r e}^{+}=\prod_{\gamma \in \Delta_{+}^{r e}, \gamma<\delta}^{\rightarrow} \exp _{q_{\gamma}}\left(a(\gamma) \xi_{\gamma} \otimes \xi_{-\gamma}\right), \\
\mathcal{R}_{r e}^{-}=\prod_{\gamma \in \Delta_{+}^{r e}, \gamma>\delta} \exp _{q_{\gamma}}\left(a(\gamma) \xi_{\gamma} \otimes \xi_{-\gamma}\right), \\
\mathcal{R}_{i m}=\exp \left(\sum_{n \geq 0} \sum_{i, j=1}^{r} c_{i, j}^{n} x_{i} \otimes x_{-j}\right) \cdot q^{(c \otimes d+d \otimes c)}
\end{gathered}
$$

and

$$
\left(c^{n}\right)_{i, j}=\left(q-q^{-1}\right) \cdot\left(\frac{\left[n\left(\alpha_{k}, \alpha_{l}\right)\right]_{q}}{n}\right)_{i, j}^{-1}
$$

for $n>0$ and

$$
\left(c^{0}\right)_{i, j}=\log q \cdot\left(\left(\alpha_{k}, \alpha_{l}\right)\right)^{-1}{ }_{i, j}
$$

The decomposition (2.19) produces the decomposition of the universal $R$-matrix $R$ for $U^{\prime}(\hat{g})$ :

$$
R=R^{+} R^{0} R^{-}
$$

where $R^{ \pm}=\mathcal{R}_{r e}^{ \pm}$and $R^{0}=\mathcal{R}_{i m} \cdot q^{-(c \otimes d+d \otimes c)}$.

In the following we use the notation of "finite vacua" representation of $U^{\prime}{ }_{q}(\hat{g})$ for a representation $V$ of $U^{\prime}{ }_{q}(\hat{g})$ whose restriction to $U_{q}(g)\left(U_{q}(g)\right.$ is generated be $e_{ \pm \alpha_{i}}, k_{\alpha_{i}}^{ \pm 1}$, $i=1, \ldots r)$ is a highest weight module generated by highest weight vector $v_{0}$.

We say that a vector $v \in V$ has a weight $\lambda(v) \in h^{*}$ ( $h$ is Cartan subalgebra of $g$ ) if $k_{\alpha_{i}} v= \pm q^{(\lambda(v), \alpha)} v$ for $i=1,2, \ldots, r$. There is a natural partial ordering in the space $h^{*}$ of weights: $\lambda<\mu$ if $\left(\mu-\lambda, \alpha_{i}\right) \in \mathbf{N}, i=1, \ldots r$. This partial ordering induces a partial ordering for weight vectors in finite vacua representations of ${U^{\prime}}_{q}(\hat{g}): v<u \Leftrightarrow \lambda(u)<\lambda(v)$. We assume also that a finite vacua representation $V$ is equiped with a basis of weight vectors $v_{i}, i=0,1, \ldots$.

For a given finite vacua representation $V$ of $U^{\prime}{ }_{q}(\hat{g})$ by means of the grading automorphism $D_{z}: U^{\prime}{ }_{q}(\hat{g}) \rightarrow U^{\prime}{ }_{q}(\hat{g})$ :

$$
D_{z}\left(e_{ \pm \alpha_{i}}\right)=z^{ \pm \delta_{i, 0}} e_{ \pm \alpha_{i}}, \quad D_{z}\left(k_{\alpha_{i}}\right)=k_{\alpha_{i}}
$$

one can define an evaluation representation (in homogenious gradation) $V(z)$ of $U^{\prime}{ }_{q}(\hat{g})$ : $\rho_{V(z)}=\rho_{V} D_{z}$.

The following theorem is a direct consequence of the presentation (2.25) of universal $R$-matrix for $U_{q}^{\prime}(\hat{g})$. 
Theorem 2.1 For any two finite vacua representations $V$ and $W$ the decomposition (2.25) produces generalized Gauss decomposition of trigonometric $R$-matrix in $V(x) \otimes$ $W(y)$ :

$$
\begin{gathered}
\rho_{V W(z)}(R)=\rho_{V W(z)}\left(R^{+}\right) \rho_{V W(z)}\left(R^{0}\right) \rho_{V W(z)}\left(R^{-}\right), \\
\rho_{V W(z)}=\rho_{V(x)} \otimes \rho_{W(y)}, \quad z=x / y,
\end{gathered}
$$

with

$$
\begin{gathered}
\rho_{V W(z)}\left(R^{+}\right)=1+\sum_{v_{i} \leq v_{j}, v_{k} \leq v_{l},(i, j) \neq(k, l)} a_{i j, k l}(z) e_{i, j} \otimes e_{k, l}, \\
\rho_{V W(z)}\left(R^{-}\right)=1+\sum_{v_{i} \geq v_{j}, v_{k} \geq v_{l},(i, j) \neq(k, l)} a_{i j, k l}(z) e_{i, j} \otimes e_{k, l}, \\
\rho_{V W(z)}\left(R^{0}\right)=\sum_{\lambda\left(v_{i}\right)=\lambda\left(v_{j}\right), \lambda\left(v_{k}\right)=\lambda\left(v_{l}\right)} a_{i j, k l}(z) e_{i, j} \otimes e_{k, l}
\end{gathered}
$$

where the r.h.s. of (2.2Y)-(2.29) are analitical functions of $z$ in the neibourhood of 0.

The dependence of $R$ from $z$ is given by an action of $D_{z} \otimes 1$ on the factors of $R$ in (2.25).

Corollary 2.1 Let all the weight spaces of $V$ and $W$ are one dimensional (as it takes place for $\mathrm{sl}_{2}$, for instance).

Then the decomposition (2.20) is exactly the Gauss decomposition of trigonometric $R$-matrix in $V(x) \otimes W(y)$.

\section{Finite vacua representations of $U_{q}^{\prime}\left(\widehat{s l}_{2}\right)$}

Let us first apply the procedure (2.2)-(2.9) of the construction of the Cartan-Weyl basis for the algebra $U_{q}\left(\widehat{s l}_{2}\right)$.

Let $\alpha$ and $\beta=\delta-\alpha$ are simple roots for the affine algebra $\widehat{s l_{2}}$ then $\delta=\alpha+\beta$ is a minimal imaginary root. We fix the following normal ordering in $\Delta_{+}$:

$$
\alpha, \alpha+\delta, \alpha+2 \delta \ldots, \delta, 2 \delta, \ldots, \ldots, \beta+2 \delta, \beta+\delta, \beta
$$

( another normal ordering is an inverse to (3.1)). Following (2.2)-(2.9) we put

$$
\begin{gathered}
e_{\delta}^{\prime}=e_{\delta}=\left[e_{\alpha}, e_{\beta}\right]_{q} \\
e_{\alpha+l \delta}=(-1)^{l}\left([(\alpha, \alpha)]_{q}\right)^{-l}\left(a d e^{\prime}\right)^{l} e_{\alpha} \\
e_{\beta+l \delta}=\left([(\alpha, \alpha)]_{q}\right)^{-l}\left(a d e^{\prime}{ }_{\delta}\right)^{l} e_{\beta} \\
e^{\prime}{ }_{l \delta}=\left[e_{\alpha+(l-1) \delta}, e_{\beta}\right]_{q}
\end{gathered}
$$


and, finally,

$$
\left(q-q^{-1}\right) E(z)=\log \left(1+\left(q-q^{-1}\right) E^{\prime}(z)\right)
$$

where $E(z)$ and $E^{\prime}(z)$ are generating functions for $e_{n \delta}$ and for $e_{n \delta}^{\prime}$ :

$$
\begin{aligned}
E(z) & =\sum_{n \geq 1} e_{n \delta} z^{-n}, \\
E^{\prime}(z) & =\sum_{n \geq 1} e_{n \delta}^{\prime} z^{-n} .
\end{aligned}
$$

The negative root vectors are given by the rule $e_{-\gamma}=e_{\gamma}^{*}$.

It is well known [0] that there exists natural algebra epimorphism $U^{\prime}{ }_{q}\left(\widehat{s} l_{2}\right) \rightarrow U_{q}\left(s l_{2}\right)$ :

$$
e_{\alpha} \rightarrow e_{\alpha}, \quad e_{\delta-\alpha} \rightarrow e_{-\alpha}
$$

and thus any highest weight representation $V$ of $U_{q}\left(s l_{2}\right)$ can be extended to a finite vacua representation $V(x)$ of $U_{q}^{\prime}\left(\widehat{s l}_{2}\right)$. Let $V_{\lambda}$ be Verma module for $U_{q}\left(s l_{2}\right)$ with a highest weight $\lambda$ and highest weight vector $v_{0}$. The action of generators $e_{ \pm \alpha}$ and $k_{\alpha}$ of $U_{q}\left(s l_{2}\right)$ can be given in the basis $v_{k}, k \geq 0$ by the relations

$$
\begin{gathered}
e_{\alpha}\left(v_{k}\right)=[k]_{q}[\lambda-k]_{q} v_{k-1}, \quad e_{-\alpha}\left(v_{k}\right)=v_{k+1}, \\
k_{\alpha}\left(v_{k}\right)=q^{\lambda-2 k} v_{k},
\end{gathered}
$$

or, in matrix notations,

$$
\begin{gathered}
e_{\alpha}=\sum_{k \geq 0}[k]_{q}[\lambda-k]_{q} e_{k-1, k}, \quad e_{-\alpha}=\sum_{k \geq 0} e_{k+1, k} \\
k_{\alpha}=\sum_{k \geq 0} q^{\lambda-2 k} e_{k, k} .
\end{gathered}
$$

Let $V_{\lambda}(-x)$ be corresponding finite vacua representation of $U^{\prime}{ }_{q}\left(\widehat{s}_{2}\right)$ (we put the sign just for convenience of further notations). According to (3.7), the structure of $V_{\lambda}(-x)$ is completely defined by (3.10) $-(3.11)$ and the relation

$$
e_{\beta}=-x e_{\alpha}
$$

. One can easily check from (3.2) that the action of generators $e_{ \pm \delta}$ is given by the formula

$$
e_{ \pm \delta}=x^{ \pm 1} \sum_{k \geq 0}\left(q^{\mp 2}[k]_{q}[\lambda-k+1]_{q}-[k+1]_{q}[\lambda-k]_{q}\right) e_{k, k}
$$

and by (3.3)-(3.5) we have

$$
\begin{gathered}
e_{\alpha+m \delta}=x^{m} \sum_{k \geq 0} q^{-\lambda+2 m-2}[k]_{q}[\lambda-k]_{q} e_{k-1, k}, \\
e_{\beta+m \delta}=-x^{m+1} \sum_{k \geq 0} q^{-\lambda+2 m} e_{k+1, k},
\end{gathered}
$$


and

$$
\begin{aligned}
e^{\prime}{ }_{m \delta}= & x^{m} \sum_{k \geq 0}\left(q^{(-\lambda+2 k-2)(m-1)-2}[k]_{q}[\lambda-k+1]_{q}-\right. \\
& \left.-q^{(-\lambda+2 k)(m-1)}[k+1]_{q}[\lambda-k]_{q}\right) e_{k, k}
\end{aligned}
$$

The relations (3.13)-(3.13) may be rewritten in a more compact form:

$$
\begin{gathered}
e_{\alpha+m \delta}=x^{m} k_{\alpha}^{-m} e_{\alpha}, \\
e_{\beta+m \delta}=-e_{-\alpha} x^{m+1} k_{\alpha}^{-m},
\end{gathered}
$$

and also

$$
\begin{gathered}
e_{-(\alpha+m \delta)}=e_{-\alpha} x^{-m} k_{\alpha}^{m}, \\
e_{-(\beta+m \delta)}=-x^{-(m+1)} k_{\alpha}^{m} e_{\alpha}
\end{gathered}
$$

Then we should write down the generating function $E^{\prime}(z)=\sum e_{m \delta}^{\prime} z^{-m}$ :

$$
\begin{gathered}
E^{\prime}(z)=\sum_{m \geq 1} \sum_{k \geq 0} x^{m} z^{-m}\left(q^{(-\lambda+2 k-2)(m-1)-2}[k]_{q}[\lambda-k+1]_{q}-\right. \\
\left.-q^{(-\lambda+2 k)(m-1)}[k+1]_{q}[\lambda-k]_{q}\right) e_{k, k}
\end{gathered}
$$

and take the logarithm of $1+\left(q-q^{-1}\right) E^{\prime}(z)$. Formal elementary manipulations with logarithms give the following answer:

$$
\begin{gathered}
\left(q-q^{-1}\right) E(z)=\log \left(1-q^{-2} k_{\alpha}^{-1} x / z\right)+\log \left(1-k_{\alpha}^{-1} x / z\right)- \\
-\log \left(1-q^{\lambda} x / z\right)-\log \left(1-q^{-\lambda-2} x / z\right)
\end{gathered}
$$

and thus the action of the imaginary root vectors is given by the following formulas:

$$
\begin{aligned}
& e_{m \delta}=\frac{q^{\lambda m}+q^{(-\lambda-2) m}-k_{\alpha}^{-m}-q^{-2 m} k_{\alpha}^{-m}}{m\left(q-q^{-1}\right)} x^{m} \\
& e_{-m \delta}=\frac{-q^{-\lambda m}-q^{(\lambda+2) m}+k_{\alpha}^{m}+q^{2 m} k_{\alpha}^{m}}{m\left(q-q^{-1}\right)} x^{-m}
\end{aligned}
$$

\section{$4 R$-matrix in tensor product of $U_{q}\left(s l_{2}\right)$-Verma mod- ules}

The universal $R$-matrix for $U_{q}\left(\widehat{s l}_{2}\right)$ has the following form KT2]:

$$
\begin{aligned}
\mathcal{R}= & \left(\prod_{n \geq 0}^{\rightarrow} \exp _{q_{\alpha}}\left(\left(q-q^{-1}\right) e_{\alpha+n \delta} \otimes e_{-\alpha-n \delta}\right)\right) . \\
& \exp \left(\sum_{n>0}\left(q-q^{-1}\right) \frac{n\left(e_{n \delta} \otimes e_{-n \delta}\right)}{[n(\alpha, \alpha)]_{q}}\right) .
\end{aligned}
$$




$$
\left(\prod_{n \geq 0}^{\leftarrow} \exp _{q_{\alpha}}\left(\left(q-q^{-1}\right) e_{\beta+n \delta} \otimes e_{-\beta-n \delta}\right)\right) \cdot \mathcal{K}
$$

where the order on $\mathrm{n}$ is direct in the first product and it is inverse in the second one. Factor $\mathcal{K}$ is defined by the formula:

$$
\mathcal{K}=q^{\frac{h_{\alpha} \otimes h_{\alpha}}{(\alpha, \alpha)}} \cdot q^{(c \otimes d+d \otimes c)}
$$

Applying the transformations (2.16) $-(2.18)$ we obtain from (4.1) the following expression for the universal $R$-matrix for $U_{q}^{\prime}\left(s_{2}\right)$ :

$$
\begin{aligned}
& R=\left(\prod_{n \geq 0}^{\rightarrow} \exp _{q_{\alpha}}\left(\left(q-q^{-1}\right) e_{\alpha+n \delta} \otimes e_{-\alpha-n \delta}\right)\right) . \\
& q^{\frac{h_{\alpha} \otimes h_{\alpha}}{(\alpha, \alpha)}} \cdot \exp \left(\left(q-q^{-1}\right) \sum_{n>0} \frac{n\left(e_{n \delta} \otimes e_{-n \delta}\right)}{[n(\alpha, \alpha)]_{q}}\right) . \\
& \left(\prod_{n \geq 0}^{\leftarrow} \exp _{q_{\alpha}}\left(\left(q-q^{-1}\right) e_{\beta+n \delta} k_{\alpha}^{-1} \otimes k_{\alpha} e_{-\beta-n \delta}\right)\right) .
\end{aligned}
$$

Let now $V_{\lambda}$ and $W_{\mu}$ be $U_{q}\left(s l_{2}\right)$-Verma modules (or their factormodules) with highest weights $\lambda$ and $\mu$. Substituting expression (3.16)-(3.19) and (3.22)-(3.23) into (4.3) we obtain the following expression for the $R$-matrix $R_{V W}(z)$ in $V_{\lambda}(x) \otimes W_{\mu}(y)(z=x / y)$ :

$$
R_{V W}(z)=R_{V W}^{+}(z) R_{V W}^{0}(z) R_{V W}^{-}(z)
$$

where

$$
\begin{gathered}
R_{V W}^{+}(z)=\left(\prod_{n \geq 0}^{\rightarrow} \exp _{q^{-2}}\left(\left(q-q^{-1}\right) z^{n} k_{\alpha}^{-n} e_{\alpha} \otimes e_{-\alpha} k_{\alpha}^{n}\right)\right) \\
R_{V W}^{-}(z)=\left(\prod_{n \geq 1}^{\leftarrow} \exp _{q^{-2}}\left(\left(q-q^{-1}\right) z^{n} e_{-\alpha} k_{\alpha}^{-n} \otimes k_{\alpha}^{n} e_{\alpha}\right)\right) \\
R_{V W}^{0}(z)=q^{\frac{h_{\alpha} \otimes h_{\alpha}}{2}} \cdot \\
\exp \left(\sum_{n>0} \frac{z^{n}}{n} \frac{\left(q^{\lambda n}+q^{(-\lambda-2) n}-k_{\alpha}^{-n}-q^{-2 n} k_{\alpha}^{-n}\right) \otimes\left(-q^{-\mu n}-q^{(\mu+2) n}+k_{\alpha}^{n}+q^{2 n} k_{\alpha}^{n}\right)}{q^{2 n}-q^{-2 n}}\right)
\end{gathered}
$$

Let $u$ be the following diagonal matrix in $V_{\lambda} \otimes W_{\mu}$ :

$$
u=z \cdot k_{\alpha} \otimes k_{\alpha}^{-1}
$$

and

$$
v_{+}=\left(q-q^{-1}\right) e_{\alpha} \otimes e_{-\alpha}, \quad v_{-}=\left(q-q^{-1}\right) e_{-\alpha} \otimes e_{\alpha} .
$$

Then the expansion of rhs of (4.4) over the powers of $v_{+}$looks as follows $\left(p=q^{-2}\right)$ :

$$
R_{V W}^{+}(z)=1+\sum_{k \geq 0}(u p)^{k} v_{+}+\frac{1}{(2)_{p}} \sum_{k \geq 0}(k+1)_{p}(u p)^{k} v_{+}^{2}+
$$




$$
+\ldots \frac{1}{(n)_{p} !} \sum_{k \geq 0} \frac{(k+1)_{p} \cdots(k+n-1)_{p}}{(n-1)_{p} !}(u p)^{k} v_{+}^{k}+\ldots
$$

or, in other terms,

$$
\begin{gathered}
R_{V W}^{+}(z)=1+\frac{1}{1-u p} v_{+}+\frac{1}{(2)_{p}} \cdot \frac{1}{(1-u p)\left(1-u p^{2}\right)} v_{+}^{2}+ \\
\ldots+\frac{1}{(n)_{p} !} \cdot \frac{1}{(1-u p) \cdots\left(1-u p^{n}\right)} v_{+}^{n}+\ldots
\end{gathered}
$$

One can interprete an expansion (4.9) as (ordered) $p$-exponent:

$$
R_{V W}^{+}(z)=: \exp _{p} \frac{q-q^{-1}}{1-u p} \cdot\left(e_{\alpha} \otimes e_{-\alpha}\right):
$$

if $: \exp _{t} a(p) \cdot b(p):$ means

$$
\begin{gathered}
: \exp _{t} a(p) b(p):=1+a(p) b(p)+\frac{1}{(2)_{t} !} a(p) a\left(p^{2}\right) \cdot b(p) b\left(p^{2}\right)+ \\
\ldots+\frac{1}{(n)_{t} !} a(p) \cdots a\left(p^{n}\right) \cdot b(p) \cdots b\left(p^{n}\right)+\ldots
\end{gathered}
$$

Analogously,

$$
\begin{gathered}
R_{V W}^{-}(z)=1+\frac{u p}{1-u p} v_{-}+\frac{p}{(2)_{p}} \cdot \frac{u p}{(1-u p)} \frac{u p^{2}}{\left(1-u p^{2}\right)} v_{-}^{2}+ \\
\ldots+\frac{p^{\frac{n(n-1)}{2}}}{(n)_{p} !} \cdot \frac{u p}{(1-u p)} \cdots \frac{u p^{n}}{\left(1-u p^{n}\right)} v_{-}^{n}+\ldots
\end{gathered}
$$

or

$$
R_{V W}^{-}(z)=: \exp _{p^{-1}} \frac{\left(q-q^{-1}\right) u p}{1-u p} \cdot\left(e_{-\alpha} \otimes e_{\alpha}\right):
$$

The expression (4.6) for $R_{V W}^{0}(z)$ we can also transform in the following way:

$$
R_{V W}^{0}(z)=f_{\lambda, \mu}(z) \cdot q^{\frac{h_{\alpha} \otimes h_{\alpha}}{2}} \cdot R_{V W}^{\left({ }^{\prime}\right)}(z) \cdot R_{V W}^{\left({ }^{\prime \prime}\right)}(z)
$$

where

$$
\begin{gathered}
f_{\lambda, \mu}(\lambda)=\exp \sum_{n \geq 1}\left(\left(q-q^{-1}\right) \frac{[\lambda n]_{q}[\mu n]_{q}}{[2 n]_{q}}\right) \frac{z^{n}}{n} \\
R_{V W}^{(\prime)}(z)=\exp \sum_{n \geq 1}\left(\left(q^{-\lambda n}-k_{\alpha}^{-n}\right) \otimes q^{-n} \frac{[\mu n]_{q}}{[n]_{q}}+q^{n} \frac{[\lambda n]_{q}}{[n]_{q}} \otimes\left(k_{\alpha}^{n}-q^{\mu n}\right)\right) \frac{z^{n}}{n}
\end{gathered}
$$

and

$$
R_{V W}^{(\prime \prime)}(z)=\exp \sum_{n \geq 1}\left(\frac{q^{n}+q^{-n}}{\left(q^{n}-q^{-n}\right)} \cdot\left(q^{-\lambda n}-k_{\alpha}^{-n}\right) \otimes\left(k_{\alpha}^{n}-q^{\mu n}\right)\right) \frac{z^{n}}{n}
$$




\section{Discussions}

Let us observe the formulas for $R$-matrix in tensor product of finite vacua representations $V_{\lambda}(x) \otimes W_{\mu}(y)$ of $U_{q}^{\prime}\left(\widehat{s l}_{2}\right)$. We present $R$ in a form of Gauss decomposition

$$
R=R_{V W}^{+}(z) \cdot R_{V W}^{0}(z) \cdot R_{V W}^{-}(z)
$$

with $R_{V W}^{ \pm}(z)$ given by expressions (4.9) and (4.12) and $R_{V W}^{0}(z)$ given by (4.14)-(4.17). One can easily see that only finite number of summands of rhs seria (4.9) and (4.12) act on a fixed weight vector of $V_{\lambda} \otimes W_{\mu}$ and the matrix coefficients of $R_{V W}^{ \pm}(z)$ are rational functions of $z=x / y$ with coefficients depending on weights. The number of poles of these coefficients is unbounded for Verma modules and bounded for finite dimensional representations (the formulas are valid for all finite vacua representations).

The structure of $R_{V W}^{0}(z)$ is more trancendental. We express $R_{V W}^{0}(z)$ as a product

$$
R_{V W}^{0}(z)=f_{\lambda, \mu}(z) \cdot q^{\frac{h_{\alpha} \otimes h_{\alpha}}{2}} \cdot R_{V W}^{\left({ }^{\prime}\right)}(z) \cdot R_{V W}^{\left({ }^{\prime \prime}\right)}(z)
$$

with the factors given by 4.15$)-(4.17)$. The scalar factor $f_{\lambda, \mu}(z)$ comes from nonlinear equation

$$
(\Delta \otimes I d) \mathcal{R}=\mathcal{R}^{13} \mathcal{R}^{23}
$$

which distinguish $R$-matrices originating from the universal $R$-matrix from other solutions of the Yang-Baxter equations. An equation (5.1) is an origin of the following functional equation on $f_{\lambda, \mu}(z)$ [Dr3]:

$$
\frac{f_{\lambda, \mu}\left(q^{2} z\right)}{f_{\lambda, \mu}\left(q^{-2} z\right)}=\frac{\left(1-q^{(\lambda-\mu)} z\right)\left(1-q^{(\mu-\lambda)} z\right)}{\left(1-q^{(\lambda+\mu)} z\right)\left(1-q^{-(\mu+\lambda)} z\right)}
$$

and express $f_{\lambda, \mu}(z)$ in terms of $q$-gamma functions (for rational variant see [S]) or, more conveniently, in terms of infinite products $(z ; q)_{\infty}$ GR, where

$$
\begin{gathered}
(z ; q)_{\infty}=\prod_{k=0}^{\infty}\left(1-z q^{k}\right): \\
f_{\lambda, \mu}(z)=\frac{\left(z q^{\lambda-\mu-2} ; q^{-4}\right)\left(z q^{\mu-\lambda-2} ; q^{-4}\right)}{\left(z q^{\lambda+\mu-2} ; q^{-4}\right)\left(z q^{-\lambda-\mu-2} ; q^{-4}\right)}
\end{gathered}
$$

Presentation (5.2) has sense for any $q \neq 1$ since the sum of weights in nominator and denominator of r.h.s. of (5.2) coincide.

Such a function was used in [FR as a simplest solution of $q$-difference KnizhnikZamolodchikov equation. The diagonal matrices $R_{V W}^{\left({ }^{\prime}\right)}(z)$ and $R_{V W}^{\left({ }^{\prime \prime}\right)}(z)$ have rational entries for integer weights $\lambda$ and $\mu$. For instance, one can easily see that for $R_{V W}^{\left({ }^{\prime}\right)}(z)$ if notices that the coefficients $\frac{[\lambda n]_{q}}{[n]_{q}}$ are finite polinomials (geometric progressions) in this case. But for noninteger weights $\lambda$ and $\mu$ the entries of $R_{V W}^{\left({ }^{\prime}\right)}(z)$ and $R_{V W}^{\left({ }^{\prime \prime}\right)}(z)$ are also can be expressed only in terms of $q$-gamma functions analogously to (5.2). This means that for generic Verma modules $V_{\lambda}$ and $W_{\mu}$ the trigonometric $R$ matrix in $V_{\lambda}(x) \otimes W_{\mu}(y)$ cannot be expressed as a matrix with entries of rational functions. However, the formulas (4.15) - 4.17) give possibility to express easily all the matrix coefficients in terms of basic functions $(z ; q)_{\infty}$. Note that there is an analogous presentation for rational $R$-matrices. It is discussed in the forthcoming paper. 


\section{References}

[AST] Asherova, R.M., Smirnov, Yu.F., and Tolstoy, V.N. A description of some class of projection operators for semisimple complex Lie algebras. Matem. Zametki 26 (1979), $15-25$.

[Ch1] Cherednik, I.V., A new interpretation of Gelfand-Zetlin bases. Duke Math. J. 54 (1987), 563-577.

[Ch2] Cherednik, I.V., Quantum groups as hidden symmetries of classical representation theory. in "Differential geometric methods of theoretical Physics" (A.I.Solomon Ed.), World Scientific, Singapore (1989), 47-54.

[Dr1] Drinfeld, V.G. A new realization of Yangians and quantized affine algebras. Soviet Math. Dokl. 32 (1988), 212-216.

[Dr2] Drinfeld, V.G. Quantum groups. Proc. ICM-86 (Berkely USA) vol.1, 798-820. Amer. Math. Soc. (1987).

[Dr3] Drinfeld, V.G. Hopf algebras and the quantum Yang-Baxter equation. . Soviet Math. Dokl. 32 , (1985), 254-258.

[FR] Frenkel, I.B., and Reshetikhin, N.Yu. Quantum Affine Algebras and Holonomic Difference equations. Commun. Math. Phys., 146 (1992), 1-60.

[GR] Gasper, G., and Rahman, M. Basic Hypergeometric Series. Cambridge Univ. Press (1990).

[J1] Jimbo, M. Quantum R-matrix for the generalized Toda system. Comm. Math. Phys. 102 (1986), 537-547.

[J2] Jimbo, M. A $q$-difference analogue of $U(g)$ and the Yang-Baxter equation. Lett. Math. Phys. 10 (1985), 63-69.

[KS] Kulish, P.P. and Sklyanin, E.K. Quantum spectral transform method: recent developments. in "Integrable quantum field theories", LN in Physics 151 (1982), 61-119.

[KRS] Kulish, P.P., Reshetikhin, N.Yu. and Sklyanin, E.K. Yang-Baxter Equation and Representation Theory I. Let.Math.Phys. 5 (1981), 393-403.

[KT1] Khoroshkin, S.M., and Tolstoy, V.N. Universal R-matrix for quantized (super)algebras. Commun. Math. Phys. 141 (1991), 599-617.

[KT2] Khoroshkin, S.M., and Tolstoy, V.N. The Universal $R$-matrix for quantum nontwisted affine Lie algebras. Funkz. Analiz i ego pril. 26:1 (1992), 85-88.

[KT3] Khoroshkin, S.M., and Tolstoy, V.N. The Uniqueness Theorem for the Universal R-matrix. Lett. Math. Phys., 24 (1992), 231-244. 
[S] Smirnov, F.A. Dinamical symmetries of massive integrable models J. of Modern Phys. A, 7 suppl. $1 B$ (1992), 813-838.

[ZG] Zhang, Y-Z., and Gould, M.D., On Universal $R$-matrix for quantized nontwisted rank 3 affine Lie algebras. Lett. Math. Phys. 29 (1993), 19. 